\title{
On Transmuted Four Parameters Generalized Log-Logistic Distribution
}

\author{
Femi Samuel Adeyinka, Akintayo Kehinde Olapade* \\ Department of Mathematics, Obafemi Awolowo University, Ile-Ife, Nigeria \\ Email address: \\ fs.adeyinka247@gmail.com (F. S. Adeyinka), akolapad@oauife.edu.ng (A. K. Olapade) \\ ${ }^{*}$ Corresponding author
}

\section{To cite this article:}

Femi Samuel Adeyinka, Akintayo Kehinde Olapade. On Transmuted Four Parameters Generalized Log-Logistic Distribution. International Journal of Statistical Distributions and Applications. Vol. 5, No. 2, 2019, pp. 32-37. doi: 10.11648/j.ijsd.20190502.12

Received: May 5, 2019; Accepted: June 5, 2019; Published: July 16, 2019

\begin{abstract}
In this article we transmute the four parameters generalized log-logistic distribution using quadratic rank transmutation map to develop a transmuted four parameters generalized log-logistic distribution. The quadratic rank transmutation map helps to introduce extra parameter into the baseline distribution to enhance more flexibility in the analysis of data in various disciplines such as reliability analysis in engineering, survival analysis, medicine, biological sciences, actuarial science, finance and insurance. The mathematical properties such as moments, quantile, mean, median, variance, skewness and kurtosis of this distribution are discussed. The reliability and hazard functions of the four parameters generalized log-logistic distribution are obtained. The probability density functions of the minimum and maximum order statistics of the four parameters generalized log-logistic distribution are established and the relationships between the probability density functions of the minimum and maximum order statistics of the parent model and the probability density functions of the four parameters generalized log-logistic distribution are considered. The parameter estimation is done by the maximum likelihood method. The flexibility of the model in statistical data analysis and its applicability is demonstrated by using it to fit relevant data. The study is concluded by demonstrating that the four parameters generalized log-logistic distribution has a better goodness of fit than its parent model. We hope this model will serve as an alternative to the existing ones in fitting positive real data.
\end{abstract}

Keywords: Log-Logistic Distribution, Reliability Function, Hazard Rate Function, Parameter Estimation, Order Statistics, Transmutation

\section{Introduction}

The log-logistic distribution is very useful in the field of survival analysis. The probability density function (pdf), in its simplest form, is given by

$$
g(x)=\frac{1}{(1+x)^{2}}, x>0
$$

The distribution has been studied into some details by Shah and Dave [1], Tadikamalla and Johnson [2], O'Quigley and Struthers [3], Ragab and Green [4].

Balakrishnan, Malik and Puthenpura[5] studied log logistic model with the pdf given by

$$
g(x ; \alpha, \beta)=\frac{\beta \alpha^{\beta} x^{\beta-1}}{\left(\alpha^{\beta}+x^{\beta}\right)^{2}}, x>0, \alpha>0, \beta \geq 1
$$

where $\alpha$ is the scale parameter and $\beta$ is the shape parameter.

Olapade (2010) studied four parameters generalized log logistic distribution with pdf given by

$$
g(x)=\frac{\beta \theta \alpha^{\theta} x^{\beta-1}}{\left(\alpha+x^{\beta}\right)^{\theta+1}}, x>0, \alpha>0, \beta \geq 1, \theta>0
$$

where $\theta>0$ is the extra shape parameter with location parameter $\mu=0$. He established its properties and stated some theorems relating it to some other distributions in the literature.

Aryal [6] transmuted the log logistic model of Balakrishnan, Malik and Puthenpura [5] and obtained its mathematical properties and demonstrated its flexibility in the reliability analysis.

This research work will study the transmutation of the work of Olapade [7] and this will be referred to as 
transmuted four parameters generalized log-logistic distribution.

\section{Transmuted Four Parameters Generalized Log-logistic Distribution}

If a random variable $\mathrm{X}$ follows four parameters generalized log-logistic distribution with probability density function (pdf) given in (3) and the cumulative distribution function (cdf) given by

$$
G(x)=1-\left(\frac{\alpha}{\alpha+x^{\beta}}\right)^{\theta}, x>0 .
$$

The parameter $\alpha$ is the scale parameter, $\theta$ and $\beta$ are shape parameters. The corresponding transmuted four parameters generalized log-logistic distribution, using the quadratic rank transmutation map.

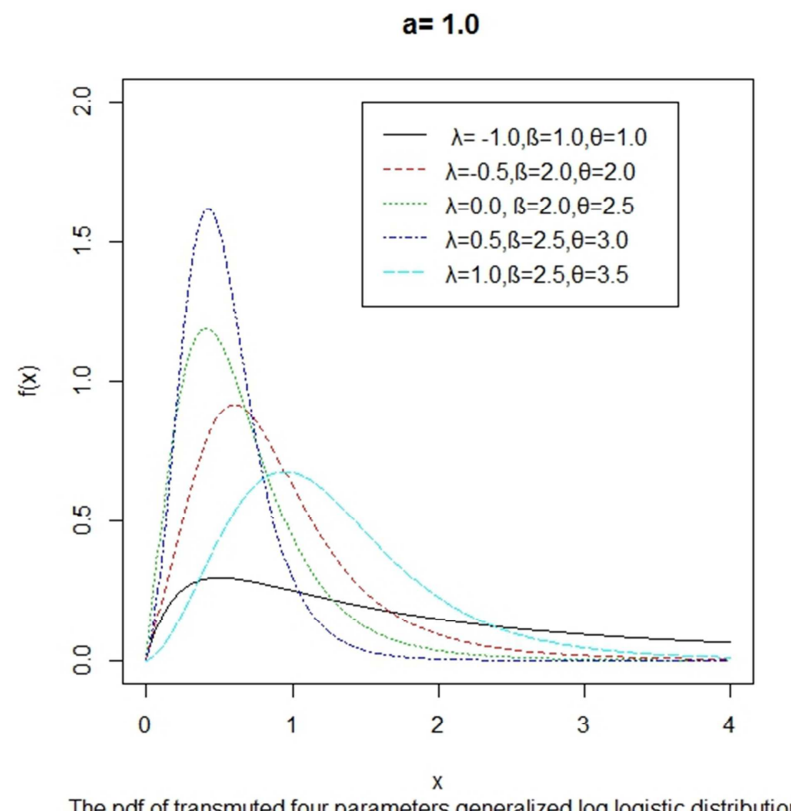

$$
F(x)=(1+\lambda) G(x)-\lambda G^{2}(x),[\lambda] \leq 1
$$

is given by

$$
F(x)=\frac{\left(\alpha+x^{\beta}\right)^{\theta}\left\{\left(\alpha+x^{\beta}\right)^{\theta}-(1-\lambda) \alpha^{\theta}\right\}-\lambda \alpha^{2 \theta}}{\left(\alpha+x^{\beta}\right)^{2 \theta}}, x>0,
$$

and the corresponding pdf is given by

$$
f(x)=\frac{\beta \theta \alpha^{\theta} x^{\beta-1}\left\{(1-\lambda)\left(\alpha+x^{\beta}\right)^{\theta}+2 \lambda \alpha^{\theta}\right\}}{\left(\alpha+x^{\beta}\right)^{2 \theta+1}}, x>0 .
$$

The parameter $\lambda$ is the transmutation parameter. We have the pdf of four parameters log-logistic distribution when parameter $\lambda=0$ in (7).

Figure 1 illustrates the graphs of pdf and cdf of transmuted four parameters generalized log-logistic distribution for different values of parameters $\alpha, \beta, \theta$ and $\lambda$.

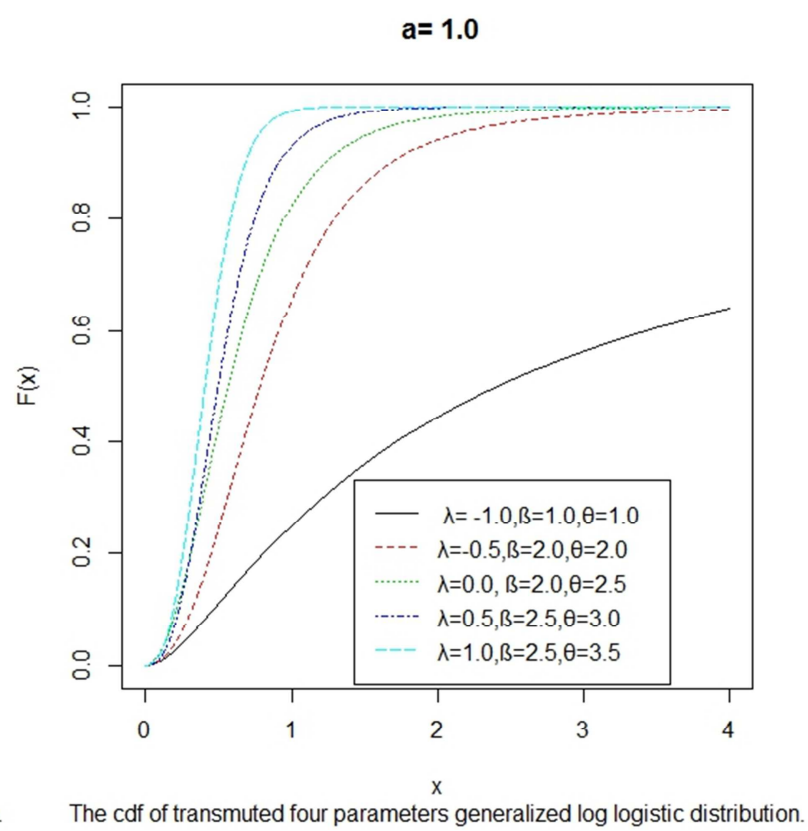

Figure 1. The graphs of pdf and cdf of transmuted four parameters generalized log-logistic distribution for different values of parameters $\alpha, \beta, \theta$ and $\lambda$.

\section{Moments and Quantile}

The $k^{\text {th }}$ order moment of transmuted four parameters generalized log-logistic random variable $\mathrm{x}$, is given by

$$
E\left[X^{k}\right]=\int_{0}^{\infty} x^{k} f(x) d x
$$

where $f(x)$ is the pdf of transmuted four parameters generalized log-logistic distribution. This is obtained as

$$
\begin{aligned}
E\left[X^{k}\right]= & \theta \alpha^{k / \beta}\{(1-\lambda) B(1+k / \beta, \theta-k / \beta)- \\
& 2 \lambda B(1+k / \beta, 2 \theta-k / \beta)\}
\end{aligned}
$$

where $B(.,$.$) is the beta function defined by$

$$
B(a, b)=\int_{0}^{1} x^{a-1}(1-x)^{b-1} d x .
$$

Using the functional relationships

$$
B(a, b)=\frac{\Gamma(a) \Gamma(b)}{\Gamma(a+b)}
$$

we have the $k^{\text {th }}$ order moments given by

$$
\begin{aligned}
E\left[X^{k}\right]=\alpha^{k / \beta} & \left\{(1-\lambda) \frac{\Gamma(1+k / \beta) \Gamma(\theta-k / \beta)}{\Gamma(\theta)}\right. \\
& \left.-\lambda \frac{\Gamma(1+k / \beta) \Gamma(2 \theta-k / \beta)}{\Gamma(2 \theta)}\right\}
\end{aligned}
$$


The first moment of the transmuted four parameters generalized log-logistic distribution which is the mean of transmuted four parameters generalized log-logistic distribution is given by

$$
\begin{aligned}
& E[X]=\alpha^{1 / \beta}\left\{(1-\lambda) \frac{\Gamma(1+1 / \beta) \Gamma(\theta-1 / \beta)}{\Gamma(\theta)}\right. \\
&\left.-\lambda \frac{\Gamma(1+1 / \beta) \Gamma(2 \theta-1 / \beta)}{\Gamma(2 \theta)}\right\}
\end{aligned}
$$

and the variance is given by

$$
\begin{aligned}
& \operatorname{var}(x)= \\
& \left\{\alpha^{2 / \beta}\left[(1-\lambda) \frac{\Gamma\left(1+{ }^{2} / \beta\right) \Gamma(\theta-2 / \beta)}{\Gamma(\theta)}-\lambda \frac{\Gamma(1+2 / \beta) \Gamma(2 \theta-2 / \beta}{\Gamma(2 \theta)}\right]\right\}- \\
& \left\{\alpha^{1 / \beta}\left[(1-\lambda) \frac{\Gamma(1+1 / \beta) \Gamma(\theta-1 / \beta)}{\Gamma(\theta)}-\lambda \frac{\Gamma(1+1 / \beta) \Gamma(2 \theta-1 / \beta)}{\Gamma(2 \theta)}\right]\right\}^{2} \text {. }
\end{aligned}
$$

The $q^{\text {th }}$ quantile $x_{q}$ of transmuted four parameters generalized log-logistic distribution is obtained from (6) as

$$
x_{q}=\left\{\alpha\left[\theta \sqrt{\frac{(1-\lambda)+\sqrt{(1+\lambda)^{2}-4 \lambda q}}{2(1-q)}}-1\right]\right\}^{1 / \beta}
$$

The median of transmuted four parameters generalized log-logistic distribution is obtained when $q=0.5$ in (9) to have

$$
x_{0.5}=\left\{\alpha\left[\theta \sqrt{(1-\lambda)+\sqrt{1+\lambda^{2}}}-1\right]\right\}^{1 / \beta}
$$

\section{Random Number Generation and Parameter Estimation}

Using the method of inversion we can generate random numbers from transmuted four parameters generalized loglogistic distribution as

$$
\frac{\left(\alpha+x^{\beta}\right)^{\theta}\left\{\left(\alpha+x^{\beta}\right)^{\theta}-(1-\lambda) \alpha^{\theta}\right\}-\lambda \alpha^{2 \theta}}{\left(\alpha+x^{\beta}\right)^{2 \theta}}=u
$$

where $u \sim U(0,1)$. After simplifying (11) we have

$$
x=\left\{\alpha\left[\theta \sqrt{\frac{(1-\lambda)+\sqrt{(1+\lambda)^{2}-4 \lambda u}}{2(1-u)}}-1\right]\right\}^{1 / \beta}
$$

Equation (12) can be used to generate random numbers when the parameters $\alpha, \beta, \theta$ and $\lambda$ are known.

\section{Parameter Estimation}

The maximum likelihood estimate of the parameters $\alpha, \beta, \theta$ and $\lambda$ that are inherent within the transmuted four parameters generalized log-logistic distribution function is given by the following.

Let $X_{1}, X_{2}, \ldots X_{n}$ be a sample of size $\mathrm{n}$ from the transmuted four parameters generalized log-logistic distribution. The likelihood function is given by

$$
\begin{gathered}
L=\beta^{n} \theta^{n} \alpha^{\theta n} \prod_{i=1}^{n} x_{i}^{\beta-1} \prod_{i=1}^{n}\left\{(1-\lambda)\left(\alpha+x_{i}^{\beta}\right)^{\theta}+\right. \\
\left.2 \lambda \alpha^{\theta}\right\} / \prod_{i=1}^{n}\left(\alpha+x_{i}^{\beta}\right)^{2 \theta+1}
\end{gathered}
$$

Hence the log-likelihood function of (11) becomes

$$
\begin{array}{r}
\ln L=n \ln \beta+n \ln \theta+n \theta \ln \alpha+(\beta-1) \sum_{i=1}^{n} \ln \left(x_{i}\right)+ \\
\sum_{i=1}^{n} \ln \left\{(1-\lambda)\left(\alpha+x_{i}^{\beta}\right)^{\theta}+2 \lambda \alpha^{\theta}\right\}-(2 \theta+
\end{array}
$$$$
\text { 1) } \sum_{i=1}^{n} \ln \left(\alpha+x_{i}^{\beta}\right) \text {. }
$$

By differentiating (14) with respect to parameters $\alpha, \beta, \theta$ and $\lambda$ and equating the result to zero we have

$$
\frac{\partial \ln L}{\partial \lambda}=\sum_{i=1}^{n} \frac{2 \alpha^{\theta}-\left(\alpha-x_{i}^{\beta}\right)^{\theta}}{\left[(1-\lambda)\left(\alpha+x_{i}^{\beta}\right)^{\theta}+2 \lambda \alpha^{\theta}\right]}=0
$$

$$
\begin{aligned}
\frac{\partial \ln L}{\partial \alpha}=\frac{n \theta}{\alpha}-(2 \theta & +1) \sum_{i=1}^{n}\left[\alpha+x_{i}^{\beta}\right]^{-1} \\
& +\theta \sum_{i=1}^{n} \frac{(1-\lambda)\left(\alpha+x_{i}^{\beta}\right)^{\theta-1}+2 \lambda \alpha^{\theta-1}}{\left[(1-\lambda)\left(\alpha+x_{i}^{\beta}\right)^{\theta}+2 \lambda \alpha^{\theta}\right]}=0
\end{aligned}
$$

$$
\begin{array}{r}
\frac{\partial \ln L}{\partial \beta}=\frac{n}{\beta}+\sum_{i=1}^{n} \ln \left(x_{i}\right)-\beta(2 \theta+1) \sum_{i=1}^{n} \frac{x_{i}^{\beta-1}}{\left(\alpha+x_{i}^{\beta}\right)}+\beta \theta(1 \\
-\lambda) \sum_{i=1}^{n} \frac{\left(\alpha+x_{i}^{\beta}\right)^{\theta} x_{i}^{\beta-1}}{\left[(1-\lambda)\left(\alpha+x_{i}^{\beta}\right)^{\theta}+2 \lambda \alpha^{\theta}\right]}=0
\end{array}
$$

$$
\begin{aligned}
\frac{\partial \ln L}{\partial \theta}=\frac{n}{\theta}-2 \sum_{i=1}^{n} & \ln \left[\alpha+x_{i}^{\beta}\right] \\
& +\sum_{i=1}^{n} \frac{2 \lambda \alpha^{\theta} \ln \alpha+\theta(1-\lambda)\left(\alpha+x_{i}^{\beta}\right)^{\theta-1}}{\left[(1-\lambda)\left(\alpha+x_{i}^{\beta}\right)^{\theta}+2 \lambda \alpha^{\theta}\right]}=0
\end{aligned}
$$

The maximum likelihood estimator $\hat{\vartheta}=(\hat{\alpha}, \widehat{\beta}, \hat{\theta}, \hat{\lambda})^{\prime}$ of parameters $\vartheta=(\alpha, \beta, \theta, \lambda)^{\prime}$ can be obtained by solving this non-linear system of equations. It is usually more convenient to use non-linear optimization algorithms such as quasiNewton algorithm to numerically maximize the loglikelihood function in (13).

\section{Reliability Analysis}

The survival function, also known as the reliability function in engineering, is the characteristic of the explanatory variable that maps a set of events, usually associated with mortality or failure of some system unto time. It is the probability that a system will survive beyond a specified time.

The transmuted four parameters generalized log-logistic distribution can be a useful model to characterize failure time of a given system because of the analytical structure. The 
reliability function $R(t)$, which is the probability of an item not failing prior to some time $t$, is defined by $R(t)=1-$ $F(t)$. The reliability function of the transmuted four parameters generalized log-logistic distribution is given by

$$
R(t)=\frac{(1-\lambda) \alpha^{\theta}\left(\alpha+t^{\beta}\right)^{\theta}+\lambda \alpha^{2 \theta}}{\left(\alpha+t^{\beta}\right)^{2 \theta}} .
$$

Figure 2 illustrates the reliability function behaviour and hazard rate function behaviour of the transmuted four parameters generalized log-logistic distribution as the value of parameter $\lambda$ varies from -1 to 1 for the selected values of parameters $\alpha, \beta$ and $\theta$ respectively.

The other characteristics of interest of a random variable is the hazard rate function also known as instantaneous failure rate defined by

$$
h(t)=\frac{f(t)}{1-F(t)}
$$

which is an important quantity characterizing life phenomenon. It can be loosely interpreted as the conditional probability of failure, given it has survived to the time $t$. The hazard rate function of transmuted four parameters generalized log-logistic distribution is given by

$$
h(t)=\frac{\beta \theta \alpha^{\theta} t^{\beta-1}\left\{(1-\lambda)\left(\alpha+t^{\beta}\right)^{\theta}+2 \lambda \alpha^{\theta}\right\}}{\left(\alpha+t^{\beta}\right)\left[(1-\lambda) \alpha^{\theta}\left(\alpha+t^{\beta}\right)^{\theta}+\lambda \alpha^{2 \theta}\right]}
$$

Note that when $t=0, h(t)=0$.

The fundamental relationship between reliability function $R(t)$ and cumulative hazard function $H(t)$ is given by $H(t)=-\ln R(t)$. The cumulative hazard function of the transmuted four parameters generalized log-logistic distribution is given by

$$
H(t)=\int_{0}^{t} h(x) d x=\ln \left\{\frac{\left(1+t^{\beta} / \alpha\right)^{2 \theta}}{\lambda+(1-\lambda)\left(1+t^{\beta} / \alpha\right)^{\theta}}\right\}
$$

It is observed that

(1) $H(t)$ is a non-decreasing for all $t \geq 0$

(2) $H(0)=0$

(3) $\lim _{t \rightarrow \infty} H(t)=\infty$.
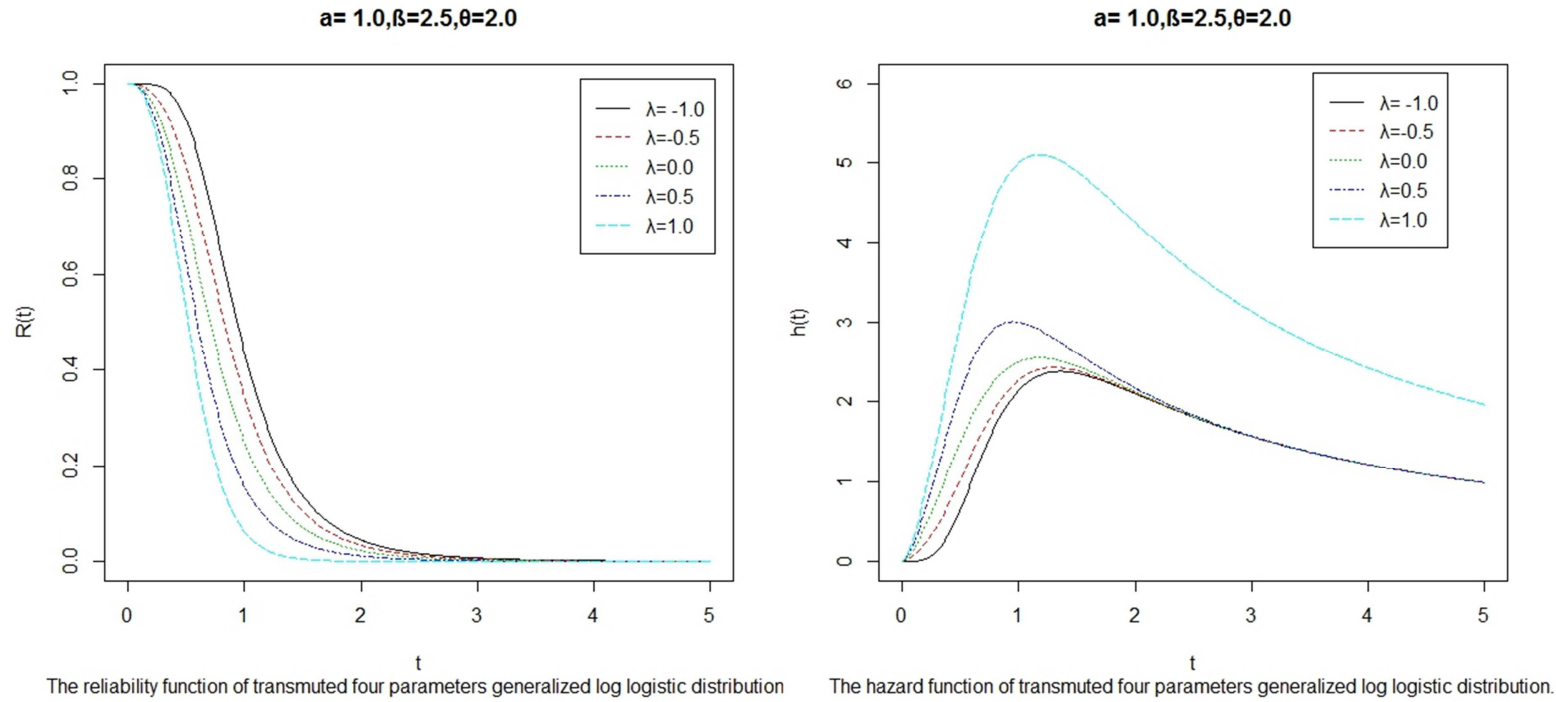

Figure 2. The graphs of reliability function and hazard function of transmuted four parameters generalized log-logistic distribution for different values of parameters $\alpha, \beta, \theta$ and $\lambda$.

\section{Order Statistics}

We know that if $X_{(1)} \leq X_{(2)} \leq \cdots \leq X_{(n)}$ denotes the order statistics of a random sample $X_{1}, X_{2} \ldots X_{n}$ from a continuous population with $\operatorname{cdf} F_{X}(x)$ and $\operatorname{pdf} f_{X}(x)$, David 1970 gave the probability density function of $X_{(r)}$ as

$$
f_{X_{(r)}}(x)=\frac{1}{B(r, n-r+1)}[F(x)]^{r-1}[1-F(x)]^{n-r} f(x)
$$

where $r=1,2, \ldots n$.

We have from (3) and (4) the pdf of the $r^{\text {th }}$ order of the four parameters generalized log-logistic random variable $X_{(r)}$ given by

$$
g_{X_{(r)}}(x)=\frac{\beta \theta \alpha^{\theta(n-r+1)} x^{\beta-1}\left\{\left(\alpha+x^{\beta}\right)^{\theta}-\alpha^{\theta}\right\}^{r-1}}{B(r, n-r+1)\left(\alpha+x^{\beta}\right)^{n \theta+1}}
$$

Therefore the pdf of the $n t h$ order four parameters generalized log-logistic statistic $X_{(n)}$ is given by

$$
g_{X_{(n)}}(x)=\frac{n \beta \theta \alpha^{\theta} x^{\beta-1}\left\{\left(\alpha+x^{\beta}\right)^{\theta}-\alpha^{\theta}\right\}^{n-1}}{\left(\alpha+x^{\beta}\right)^{n \theta+1}}
$$

and the pdf of the $1^{\text {st }}$ order four parameters generalized loglogistic distribution statistic $X_{(1)}$ is given by

$$
g_{X_{(1)}}(x)=\frac{n \beta \theta \alpha^{n \theta} x^{\beta-1}}{\left(\alpha+x^{\beta}\right)^{n \theta+1}}
$$


Note that in a particular case of $n=2,(17)$ yields

$$
g_{X_{(2)}}(x)=\frac{2 \beta \theta \alpha^{\theta} x^{\beta-1}\left\{\left(\alpha+x^{\beta}\right)^{\theta}-\alpha^{\theta}\right\}}{\left(\alpha+x^{\beta}\right)^{2 \theta+1}}
$$

and (18) yields

$$
\begin{gathered}
g_{X_{(1)}}(x)=\frac{2 \beta \theta \alpha^{2 \theta} x^{\beta-1}}{\left(\alpha+x^{\beta}\right)^{2 \theta+1}} \text { (20) } \begin{array}{l}
\text { the transmuted four parameters generalized log-logistic } \\
\text { distribution is given by }
\end{array} \\
f_{X_{(r)}}(x)=\frac{\beta \theta \alpha^{\theta} x^{\beta-1}\left\{(1-\lambda)\left(\alpha+x^{\beta}\right)^{\theta}+2 \lambda \alpha^{\theta}\right\}\left\{\left(\alpha+x^{\beta}\right)^{\theta}\left[\left(\alpha+x^{\beta}\right)^{\theta}-(1-\lambda) \alpha^{\theta}\right]-\lambda \alpha^{2 \theta}\right\}^{r-1}\left\{\left(\alpha+x^{\beta}\right)^{2 \theta}-\left(\alpha+x^{\beta}\right)^{\theta}\left[\left(\alpha+x^{\beta}\right)^{\theta}-(1-\lambda) \alpha^{\theta}\right]-\lambda \alpha^{2 \theta}\right\}^{n-r}}{B(r, n-r+1)\left(\alpha+x^{\beta}\right)^{2 n \theta+1}}(21)
\end{gathered}
$$

It can be observed that $\min \left(X_{1}, X_{2}\right)$ and $\max \left(X_{1}, X_{2}\right)$ in (19) and (20) are special cases of (7) for $\lambda=-1$ and $\lambda=1$ respectively.

Now we provide the distribution of the order statistics for the transmuted four parameters generalized log-logistic distribution variable. The pdf of the $r^{t h}$ order statistics for

Therefore the pdf of the largest order statistic $X_{(n)}$ is obtained when $r=n$ in (21) and it is given by

$$
f_{X_{(n)}}(x)=\frac{n \beta \theta \alpha^{\theta} x^{\beta-1}\left\{(1-\lambda)\left(\alpha+x^{\beta}\right)^{\theta}+2 \lambda \alpha^{\theta}\right)\left\{\left(\alpha+x^{\beta}\right)^{\theta}\left[\left(\alpha+x^{\beta}\right)^{\theta}-(1-\lambda) \alpha^{\theta}\right]-\lambda \alpha^{2 \theta}\right\}^{n-1}}{\left(\alpha+x^{\beta}\right)^{2 n \theta+1}}
$$

and the pdf of the smallest order statistic $X_{(1)}$ is obtained when $r=1$ in (21) and it is given by

$$
f_{X_{(1)}}(x)=\frac{\beta \theta \alpha^{\theta} x^{\beta-1}\left\{\left(\alpha+x^{\beta}\right)^{2 \theta}-\left(\alpha+x^{\beta}\right)^{\theta}\left[\left(\alpha+x^{\beta}\right)^{\theta}-(1-\lambda) \alpha^{\theta}\right]-\lambda \alpha^{2 \theta}\right\}^{n-1}}{\left(\alpha+x^{\beta}\right)^{2 n \theta+1}}
$$

\section{Application}

The data consists of 30 observations of March precipitation (in inches) in Minneapolis/St Paul. The data set is extracted from Usman, Haq and Talib [8]. The observations are:

$0.77,1.74,0.81,1.20,1.95,1.20,0.47,1.43,3.37,2.20$, $3.00,3.09,1.51,2.10,0.52,1.62,1.31,0.32,0.59,0.81,2.81$, $1.87,1.18,1.35,4.75,2.48,0.96,1.89,0.90,2.05$.

A quasi Newton algorithm was implemented in $\mathrm{R}$ package and the performances of the models are shown in Table 1. Akaike Information criterion (AIC), Corrected Akaike Information criterion (AICC) and Bayesian Information criterion (BIC) were respectively used to compare the performance of the transmuted four parameters generalized log-logistic distribution (TGLL) to its parent model (GLL) in (3).

Table 1. Performance of the models.

\begin{tabular}{llllll}
\hline Model & & L & AIC & AICC & BIC \\
\hline & $\beta=2.113$ & & & & \\
TGLL & $\alpha=1.237$ & 142.672 & 289.344 & 289.518 & 289.059 \\
& $\theta=1.256$ & & & & \\
& $\lambda=0.621$ & & & & \\
GLL & $\beta=1.921$ & & & & \\
& $\alpha=2.556$ & 147.135 & 296.270 & 296.326 & 296.127 \\
& $\theta=0.894$ & & & & \\
\hline
\end{tabular}

It is observed that the transmuted four parameters generalized log-logistic distribution (TGLL) performed better than its parent model (GLL) in (3).

\section{Conclusion}

In this article, we have introduced a new generalization of the four parameters generalized log-logistic distribution called transmuted four parameters generalized log-logistic distribution. The distribution which is generalized by using the quadratic rank transmutation map. Some mathematical properties along with estimation issues are addressed. The hazard rate function and reliability function behaviours of transmuted four parameters generalized log-logistic distribution show that the model can be used to model positive data that follow the subject model. Further studies can be done in the area of applicability of the model in various disciplines.

\section{References}

[1] Shah, B. K., and Dave, P. H. (1963). A note on log-logistic distribution. Journal of Mathematical Sciences of University of Baroda. Vol 12. Pp 21-22.

[2] Tadikamalla, P. R., and Johnson, N. L. (1982). Systems of frequency curves generated by the transformation of logistic variables. Biometrika. Vol 69. Pp 461465.

[3] O'Quigley, J., and Struthers, L. (1982). Survival model based upon the logistic and log-logistic distribution. Computer programmes in Biomedicine. Vol 15. Pp 3-12.

[4] Ragab, A., and Green, J. (1984). On order statistics from the log-logistic distribution and their properties. Communications in statistics-Theory and Methods. Vol 13. Pp 2713-2724.

[5] Balakrishnan, N., Malik, H. J., and Puthenpura, S. (1987): Best linear unbiased estimation of location and scale parameters of the log-logistic distribution. Communications in Statistics-Theory and Methods. Vol 12. Pp 3477-3495.

[6] Aryal, G. R. (2013). Transmuted log-logistic distribution. Journal of Statistics Applications and probability. 2 (1), 11-20. 
[7] Olapade, A. K. (2010). On log-logistic and a four-parameter generalized log-logistic distributions. Proceedings of the Jangjeon Mathematical Society. Vol 13. Pp 67-76.

[8] Usman, R. M, Haq, M. A and Talib, J (2017). Kumaraswamy Half-Logistic Distribution: Properties and Applications. Journal of Statistics Applications and Probability. No 3,597-609.

[9] Aryal, G. R, and Tsokos, C. P. (2009). On the transmuted extreme value distribution with application. Nonlinear Analysis: Theory, Methods and Application.71 (12), el401el407.

[10] Aryal, G. R, and Tsokos, C. P. (2011). Transmuted Weilbull distribution: A generalization of Weilbull probability distribution. European Journal of Pure and Applied Mathematics. 4 (2), 89-102.

[11] Bjerkedal, T (1960). Acquisition of Resistance in Guinea Pigs infected with Different Doses of Virulent Tubercle Bacilli, American Journal of Hygiene, 72, 130-148.

[12] Granzoto, D. C. T., Louzada, F., and Balakrishnan, N. (2017). Cubic rank transmuted distributions: Inferential issues and applications. Journal of statistical Computation and Simulation. 87: 2760-2778, doi. 101080/00949655.2017.1344239.

[13] Haq, M. A, (2016). Kumaraswamy Exponentiated Inverse Rayleigh Distribution.
[14] Merovci, F., Alizadeh, M., and Hamedani, G. (2016). Another Generalized Transmuted Family of Distributions: Properties and Applications. Austrian Journal of Statistics. 45, 71-93.

[15] Merovci, F. (2014). Transmuted Generalized Rayleigh Distribution. Journal of Statistics Applications and Probability. 3 (1), 9-20.

[16] Merovci, F., Elbatal, I. (2014). Transmuted Lindley-geometric Distribution and its Applications. Journal of Statistics Applications and Probability. 3 (1), 77-91.

[17] Merovci, F., Puka, L. (2014). Transmuted Pareto Distribution. Probstat.7, 1-11.

[18] Olapade, A. K. (2003). On the type I generalized logistic, loglogistic and generalized log-logistic distributions. Proceedings of the Jangjeon Mathematical Society. Vol 6. Pp 137-146.

[19] Rahman M. M, Al-Zahrani B, Shahbaz M. Q (2018). A general transmuted family of distributions. Pak J Stat Oper Res 14: 451-469.

[20] Shaw, W. T, and Buckley, I. R. (2009). Alchemy of Probability Distributions: Beyond Gram-Charlier and Cornish -Fisher Expansions, and Skewed- kurtotic Normal Distribution from a Rank Transmutation Map. arxivpreprint arxiv: 0901.0434. 